\title{
Clinical Characteristics of Trigeminal Neuralgia Patients at Dr. Hasan Sadikin General Hospital Bandung Indonesia in 2010-2012
}

\author{
Sri Hudaya Widihastha, ${ }^{1}$ Henny Anggraini Sadeli, ${ }^{2}$ Arifin Sunggono ${ }^{3}$ \\ ${ }^{1}$ Faculty of Medicine Universitas Padjadjaran, ${ }^{2}$ Department of Neurology Faculty of Medicine \\ Universitas Padjadjaran/Dr. Hasan Sadikin General Hospital Bandung, ${ }^{3}$ Department of Anatomy \\ and Cell Biology Faculty of Medicine Universitas Padjadjaran
}

\begin{abstract}
Background: Trigeminal neuralgia is a frequently found craniofacial neuralgia. The clinical characteristics of trigeminal neuralgia patients at Dr. HasanSadikin General Hospital, Bandung, Indonesia has never been described before. This study conducted an overview of clinical characteristics of trigeminal neuralgia to determine the characteristic features of trigeminal neuralgia.

Methods: This was a non-experimental study using secondary data with a retrospective descriptive method. Data related to medical records of 76 patients with trigeminal neuralgia was collected over a period of 2010 to 2012, treated at Dr.HasanSadikin General Hospital Bandung, Indonesia.

Results: Mean age was 57.6 years (range 11 to 84 years), no significant difference in gender (male:females $51.3 \%: 48.7 \%$ ). Chewing and swallowing was reported as triggering stimuli to 42 (55\%) patients. Two patients $(2.7 \%)$ had suffered pain on bilateral side of the face, $46(60.5 \%)$ patients the right side, and 28 patients $(36.8 \%)$ on the left side. Pain on maxillary nerve division was found in $58(76.3 \%)$ patients. Sixty one of the patients $(80 \%)$ received Carbamazepine.

Conclusions: The peak age was between the fifth and sixth decades of life with equal representation of male to female incidence. Right side and the maxillary division was found to be the most frequently site of pain. Chewing and swallowing was reported to be the most frequent triggering stimuli and most of patients received Carbamazepine as therapy. [AMJ.2017;4(1):47-51]
\end{abstract}

Keywords: Clinical characteristic, neuropathic pain, trigeminal neuralgia

\section{Introduction}

Trigeminal neuralgia is a form of neuropathic pain disorder characterized by episodes of pain in the area which innervated by the trigeminal nerve and characterized as a spontaneous, recurrent, sharp, and paroxysmal pain. ${ }^{1}$ Trigeminal neuralgia is one of craniofacial neuralgia which is known often to be found. ${ }^{1-3}$ with a prevalence of 0.1-0.2 per thousand and an incidence ranging from about 4-5/100,000/ year up to $20 / 100,000 /$ year after age $60{ }^{4}$ Trigeminal neuralgia has been classified into Type 1 and Type 2 subtypes. Type 1 is characterized by lancinating, intermittent pain present for more than $50 \%$ of the time, while type 2 is characterized by a constant pain that is present for more than $50 \%$ of the time. ${ }^{5}$

This study conducted an overview of clinical characteristics of trigeminal neuralgia to determine the characteristic features of trigeminal neuralgia. The results of this study was expected to be useful to improve the knowledge and literature on trigeminal neuralgia, which can be used as references for further research. It is also expected that the results can provide an overview of the characteristics of trigeminal neuralgia, which can be considered for early detection the disease and further management of the patient.

\section{Methods}

This was a non-experimental study using secondary data with a retrospective descriptive method. Data related to medical records of 76 patients with trigeminal neuralgia in the Outpatient Clinic of Neurology Department, Dr. Hasan Sadikin General Hospital, Bandung

Correspondence: Sri Hudaya Widihastha, Faculty of Medicine, Universitas Padjadjaran, Jalan Raya Bandung-Sumedang Km.21, Jatinangor, Sumedang, Indonesia, Phone: +62 85659966936 Email: s.widihastha@gmail.com 
was searched and collected over a period of three years, from 2010 to 2012. All available medical records of patients with trigeminal neuralgia as diagnosis are used, i.e. total sampling method. The inclusion criterion was complete medical record with trigeminal neuralgia as diagnosis, and medical records with incomplete data were excluded. The variables analyzed were age at onset, gender, site of involvement, triggering stimuli, affected divisions of trigeminal nerve, and management therapy given to the patients.

Data collection approval was obtained from the Health Research Ethics Committee of the Dr Hasan Sadikin General Hospital. The analysis was performed by using software applications Microsoft Excel and the results of this study were then evaluated and presented descriptively in table.

\section{Results}

The age of patients was ranged between 11 to 84 years old, with a mean age of 57.6 years. The peak incidence was in the fifth and sixth decades of life (Table 1).

Females comprised $51 \%$ of the patients, representing a ratio with males of 5.3:5, reflecting that female gender is more common than male with no significance difference between them.

Table 1 Clinical Characteristics of Trigeminal Neuralgia Patients

\begin{tabular}{|c|c|c|}
\hline Characteristics & $\begin{array}{c}\text { Number of patients } \\
(n=76)\end{array}$ & Percentage (\%) \\
\hline \multicolumn{3}{|l|}{ Gender } \\
\hline Male & 37 & 49 \\
\hline Female & 39 & 51 \\
\hline \multicolumn{3}{|l|}{ Age (years old) } \\
\hline Childhood (0-14) & 1 & 1 \\
\hline Adulthood (15-49) & 17 & 23 \\
\hline Elderly (>49) & 58 & 76 \\
\hline \multicolumn{3}{|l|}{ Triggering Stimuli } \\
\hline Chewing and swallowing & 42 & 55 \\
\hline Talking & 18 & 24 \\
\hline Washing & 15 & 20 \\
\hline Tooth brushing & 11 & 15 \\
\hline Wind-blowing & 4 & 5 \\
\hline Shaving & 3 & 4 \\
\hline Touching & 3 & 4 \\
\hline Opening mouth & 3 & 4 \\
\hline Heat & 1 & 1 \\
\hline \multicolumn{3}{|l|}{ Management } \\
\hline Carbamazepine & 61 & 80 \\
\hline Amitriptyline & 34 & 45 \\
\hline Tramadol & 4 & 5 \\
\hline Phenytoin & 3 & 4 \\
\hline Baclofen & 1 & 1 \\
\hline Gabapentin & 1 & 1 \\
\hline Surgical operation & 1 & 1 \\
\hline
\end{tabular}


Table 2 Affected Divisions and Site of Involvement in Trigeminal Neuralgia Patients

\begin{tabular}{lcccc}
\hline \multirow{2}{*}{$\begin{array}{c}\text { Affected divisions of trigeminal nerve } \\
\text { and site of involvement }\end{array}$} & Bilateral & \multicolumn{2}{c}{ Side of face } & \multirow{2}{*}{ Total } \\
\cline { 3 - 4 } & & Right side & Left side & \\
\hline Ophthalmic & 0 & 3 & 1 & 4 \\
Maxillary & 1 & 13 & 11 & 25 \\
Mandibular & 0 & 8 & 6 & 14 \\
Ophthalmic, Maxillary & 0 & 4 & 2 & 6 \\
Ophthalmic, Mandibular & 0 & 0 & 0 & 0 \\
Maxillary, Mandibular & 1 & 12 & 2 & 15 \\
Ophthalmic, Maxillary, Mandibular & 0 & 6 & 6 & 12 \\
Total & 2 & 46 & 28 & 76 \\
\hline
\end{tabular}

Chewing and swallowing was reported to be the most frequent triggering stimuli. All of the 76 patients received medical treatment. Sixtyone of the patients $(80 \%)$ received different dosages of carbamazepine and most of cases received more than one medical treatment. There was one case of surgical operation as other forms of modality employed to further ease the pain.

Two patients had bilateral trigeminal nerve involvement constituting $2 \%$ of the series (Table 2). Of the remaining 74 cases, 46 patients $(61 \%)$ suffered pain on the right side of the face, and $28(37 \%)$ patients on the left side. This finding gave a right site and left site ratio of 1.7:1, confirming a predominance of right side facial affliction.

The maxillary division was found to be the most frequently involved branch. Twenty-five patients (33\%) were reported having neuralgic pain only at the maxillary distribution of the face, fifteen patients $(20 \%)$ suffered the condition with the additional involvement of the mandibular division on the same side, six patients $(8 \%)$ suffered the condition with the additional involvement of the ophthalmic division, and twelve patients (16\%) were reported having neuralgic pain confined solely at all division of trigeminal nerve distribution of the face. Fourteen patients (18\%) were reported having neuralgic pain at only the mandibular distribution of the face and the least frequent divisions of trigeminal neuralgia is ophthalmic division (5\%).

\section{Discussions}

This present study reported that the peak age of the disease was between the fifth and sixth decades of life. Similar findings was also reported by previous retrospective studies which reported that the peak age of onset were between the fifth and eighth decades of life.$^{6-9}$ Loh et al. ${ }^{10}$ stated on his study that this concurrence supports the cardinal rule of subjecting patients under 40 years of age who complain of neuralgia-like pain in the face to a detailed neurological assessment to exclude associated diseases like multiple sclerosis. The causes of trigeminal neuralgia include mechanical suppression by blood vessels, arterial venous malformations surrounding, suppression by lesions or tumors, multiple sclerosis, physical damage of the trigeminal nerve due to surgery or infection, and other unknown factors. Therefore, there were unusual patients aged less than 40 years cases reported in this recent study, which needed to be investigated further.

Katusic et al. $^{6}$ reported female predominance in the ratio of 5.9:3.4. Although other reviewers also reported that the disorder was appeared having a gender inclination, different result was seen in this present study. The result reported that there was more likely an equal representation of male to female incidence, with a ratio of 5.3:5. Nayyar et al. ${ }^{9}$ reported that male predominance, was more likely due to smaller sample size. This finding may be unique to the general populations and, therefore, deserves further study with a larger sample size.

The majority of the patients in this study were sensitive to a multitude of triggering stimuli which was varied in both type and intensity. Most common triggering stimuli included chewing and swallowing (55\%), talking (24\%), washing (20\%), tooth brushing $(15 \%)$. Those triggering stimulus were also 
reported in the previous study. ${ }^{10}$

Neto et al. ${ }^{11}$ stated that in trigeminal neuralgia the right side of the face usually afflict more than the left, since it is known that the foramen rotundum and foramen ovale on the right is narrower. In this study, the right side of the face was also much more involved (ratio 1.7:1). The proportion of bilateral cases in this study (2\%) has similar range of percentage with those of previous reports which were stated as less than, or equal to 3 $\%{ }^{6}$

This study demonstrated about 58 of the patients $(76 \%)$ in the present series reported affliction of the maxillary division either alone or in combination with the other two divisions. This report made the maxillary branch as the most frequently affected branch of the fifth cranial nerve. Reports from other studies stated the similar findings. ${ }^{6,8}$ Loh et al. ${ }^{10}$ reported that contradictory result, stated mandibular divisions was the most frequently affected branch. However, all support the usual feature of this condition in which the mandibular and maxillary divisions are more commonly involved than the ophthalmic. These presentations emphasized the importance of eliminating potential dental etiology, since the pain may mimic that of toothache because of the maxillary and mandibular site of pain and it is well known that dental extractions have been performed because of wrong diagnosis. ${ }^{10}$

From this present study, the ophthalmic division involvements to the pain need to be investigated further, because the number of patients $(29 \%)$ is higher than the studies conducted before hand. Since this present study was retrospective, it is needed to consider the limitation of retrospective study and the lack of further examination, for example Magnetic Resonance Imaging (MRI) on patients. It was also known that central sensitization of neuropathic pain can affect the distributions of patients' sensation of pain, especially to the area of ophthalmic division, which is known as a very rare to be affected directly by trigeminal neuralgia.

Pharmacological treatment of trigeminal neuralgia is usually used as the first option and, in some patients surgical options may be offered. ${ }^{10}$ Carbamazepine is highly effective and specific for this condition..$^{12}$ Carbamazepine is a tricyclic imipramine with mechanism of action related to its ability to block voltage sensitive sodium channels which result in stabilization of the hyper excitable trigeminal neural membranes. ${ }^{13}$ Some previous studies and its subsequent wide-spread use of it in trigeminal neuralgia case made carbamazepine as a drug of choice. ${ }^{14}$ Four placebo-controlled studies totaling 147 patients demonstrated the efficacy of carbamazepine. ${ }^{14}$ The treatment response in these trials was robust, with 58 to $100 \%$ of patients on carbamazepine attaining complete or near complete pain control as compared to 0 to $40 \%$ of patients on placebo. ${ }^{14}$ which makes carbamazepine as the first line drug for medical management of the disease. ${ }^{12,15}$ Carbamazepine reduced both the frequency and intensity of painful paroxysms and was equally efficacious for spontaneous and trigger-evoked attacks. ${ }^{12}$ This therapeutic response also aided in the diagnostic cconfirmation of the condition in this study. ${ }^{10}$ On this present study, it was found that Carbamazepine and Phenytoin can be given as monotheraphy.

Most of the patients in the present study were treated with Carbamazepine, and they responded favourably. Possible side effects such as skin rashes, leukopenia, thrombocytopenia, abnormal liver function, and cerebellar dysfunction may occur in 5 to $10 \%$ of those patients taking these medications. ${ }^{10}$ The long term effects of Carbamazepine have been evaluated in one study, showing either loss of effect or problem with tolerability in one half patients over 10 years. Other treatments were given including tricyclic antidepressant, Amitriptyline (45\%). This antidepressant treatment was also given to the patients in the study that conducted by Loh et al. ${ }^{10}$

Some of the patients also received various medical treatment, such as Tramadol (5\%), Phenytoin (4\%), Gabapentin (1\%), and Baclofen (1\%). Those treatment were useful as an alternative in patients who need add on therapy beside carbamazepine or the given therapy is known to be ineffective. ${ }^{12,16}$ Surgical intervention was employed to relieve the intractablepain of one young-aged patient. However, it is not without risk of morbidity and mortality. ${ }^{10}$ Each surgical technique for treatmentoftrigeminalneuralgiahasmeritsand limitations and microvasculardecompression was known to provide the highest rate of longterm patient' satisfaction with the lowest rate of pain recurrence. ${ }^{17}$

Limitation in terms of time and number of samples in this study, making further research deemed necessary. Lack of specific clinical or laboratory test exists for the diagnosis of symptomatic trigeminal neuralgia might be also the limitation of this study, since the etiological basis of classic trigeminal neuralgia is unknown but vascular compression of the 
trigeminal nerve roots has emerged as the likely cause in most cases. ${ }^{19}$

As a conclusion, this study demonstrated numerous clinical similarities of trigeminal neuralgia afflicting to the previous studies. This present study reported that the peak age was between the fifth and sixth decades of life with equal representation of male to female incidence. Right side and the maxillary division were found to be the most frequently site of pain. Chewing and swallowing were reported to be the most frequent triggering stimuli and most of patients received Carbamazepine as therapy. The sample size could be increased to present clearer data evidence. Follow-up studies would be beneficial to establish distinct Indonesian patterns in this neurological disorder for further recommendation.

\section{References}

1. Prasad S, Galetta S. Trigeminal neuralgia: historical notes and current concepts. Neurologist. 2009;15(2):87-94.

2. Krafft RM. Trigeminal neuralgia. Am Fam Physician 2008;77(9):1291-6.

3. Hall GC, Carroll D, Parry D, McQuay HJ. Epidemiology and treatment of neuropathic pain: the UK primary care perspective. Pain. 2006;122(1-2):156-62.

4. Manzoni GC, Torelli P. Epidemiology of typical and atypical craniofacial neuralgias. Neurol Sci. 2005;26(2):65-7.

5. Eller JL, Raslan AM, Burchiel KJ. Trigeminal neuralgia: definition and classification. Neurosurg Focus. 2005;18(5):E3.

6. Katusic S, Beard CM, Bergstralh E, Kurland LT. Incidence and clinical features of trigeminal neuralgia, Rochester, Minnesota, 1945-1984. Ann Neurol. 1990;27(1):89-95.

7. Jainkittivong A, Aneksuk V, Langlais RP. Trigeminal neuralgia: a retrospective study of 188 Thai cases. Gerodontology. 2012;29(2):e611-7.

8. Siqueira SR, Teixeira MJ, Siqueira JT. Clinical characteristics of patients with trigeminal neuralgia referred to neurosurgery. Eur J Dent. 2009; 3(3):207-212.

9. Nayyar AS, Khan M. Trigeminal neuralgia: revisiting clinical characteristics in the
Indian scenario. Jour of Med Sc \& Tech. 2012;1(2):9-17.

10. Loh HS, Ling SY, Shanmuhasuntharam P, Zain R, Yeo JF, Khoo SP. Trigeminal neuralgia: a retrospective survey of a sample of patients in Singapore and Malaysia.Aust Dent J. 1998;43:(3):188-91.

11. Neto HS, Camilli JA, Marques MJ. Trigeminal neuralgia is caused by maxillary and mandibular nerve entrapment: greater incidence of right-sided facial symptoms is due to the foramen rotundum and foramen ovale being narrower on the right side of the cranium. Med Hypotheses. 2005;65(6):1179 - 82.

12. Gronseth G, Cruccu G, Alksne J, Argoff C, Brainin M, Burchiel K, et al. Practice parameter: the diagnostic evaluation and treatment of trigeminal neuralgia (an evidence-based review): report of thequality standards subcommittee of the American academy ofneurology and the European Federation of Neurological Societies. Neurology.2008;71(15):1183-90.

13. McNamara JO. Goodman \& Gilman's the pharmacological basis therapeutics. 12th ed. New York. McGraw Hill: 2001.

14. Campbell FG, Graham JG, Zilkha KJ. Clinical trial of Carbamazepine (Tegretol) in trigeminal neuralgia. J Neurol Neurosurg Psychiatry. 1966;29(3):265-7.

15. Jorns TP, Zakrzewska JM. Evidence-based approach to the medical management of trigeminal neuralgia. Br J Neurosurg. 2007;21(3):253 - 61.

16. Bennetto L, Patel NK, Fuller G. Trigeminal neuralgia and its management. BMJ. 2007;334(586):201-5.

17. Tatli M, Satici O, Kanpolat Y, Sindou M. Various surgical modalities for trigeminal neuralgia: literature study of respective long-term outcomes. Acta Neurochirurgica. 2008;150(3):243-255.

18. Hasan S, Khan NI, Sherwani OA, Bhatt V, Asif S. Trigeminal Neuralgia: an overview of literature with emphasis medical management. International Research Journal of Pharmacy. 2012;3(11):235-8.

19. Thomas KL, Vilensky JA. The anatomy of vascular compression in trigeminal neuralgia. Clin Anat. 2014;27(1):89-93 